\title{
Datierungsfragen am Bockstein
}

\author{
Von Robert Wetzel, Tübingen
}

\author{
Mit 5 Abbildungen im Text und einer Tafel (II)
}

Zusammenfassung. Am Bockstein im Lonetal (Württemberg) sind 1954-56 die Schichten an der Brandplatte und am Hang unterhalb der Schmiede ausgegraben und, soweit technisch möglich, bis in die Ablagerungen des Talgrundes verfolgt worden. An neu und früher ergrabenen Schichten wurden sedimentanalytische, faunistische und floristische Spezialuntersuchungen durchgeführt (Elisabeth Schmid, Ulrich Lemmann, Paul Filzer). Nach den bisher vorliegenden Ergebnissen entstand wahrscheinlich die violette Schicht des Bodksteinlochs mit ihrer Faustkeilkultur in einer ausgeprägten Wärmephase des Riss-Würm-Interglazials (alle Benennungen im klassischen Sinne gebraucht), die etwas ältere Schicht der Faustkeil-Hauptkultur der Bocksteinschmiede in einer früheren, kühleren Phase der selben Wärmezeit, oder in einer späten Phase der ausgehenden Riss-Eiszeit. Alter als die Hauptkultur der Schmiede ist ein mächtiger Stoß von Schichten, mit nochmals mehrfachem Wechsel von wärmezeitlich und kältezeitlich entstandenen Sedimenten. Die Reihe der Faustkeilkulturen vom Bockstein, vor allem die Hauptkultur der Schmiede, scheint so alt zu sein, daß in ihr nach gewissen Ausgangsformen für spätere Kulturentfaltung gesucht werden darf. In bemerkenswertem Gegensatz zu übereinstimmenden sedimentanalytischen und floristischen Befunden fehlt im Bilde der vorliegenden Säugetierreste fast jede spezifische, kälte- oder wärmezeitliche Prägung.

Ré s u mé. Au Bockstein dans la vallée de la Lone (Württemberg) les couches de la Brandplatte et du Hang situé au dessous de la Schmiede ont été mises à jour et autant que le permettaient nos moyens techniques, suivies jusqu' aux gisements au fond de la vallée. Dans les couches de découverte récente et ancienne, des recherches particulieres ont été faites, concernant l'analyse des sédiments, la faune et la flore (Elisabeth Schmid, Ulrich Lehmann, Paul Filzer). D'aprés les résultats que nous possédons jusqu'à ce jour, la couche violette du Bocksteinloch avec sa civilisation de coups de poing a pris naissance vraisemblablement dans une phase nettement caractérisée de l'interglaciaire de Riss-Würm (tous ces termes sont pris dans leur sens classique), la couche un peu plus ancienne de la civilisation principale des coups de poing de la Bocksteinschmiede, dans une phase antérieure, plus fraiche de la même époque de chaleur, ou bien dans une phase tardive de la fin de l'époque glaciaire du Riss. Plus ancien que la civilisation principale de la Schmiede est un imposant amoncellement de couches dans lesquels on voit une fois de plus alterner à plusieurs reprises les sédiments d'une époque de chaleur et ceux d'une époque de froid. La série des civilisations des coups de poing et spécialement la civilisation principale de la Schmiede, paraissent être si anciennes que l'on peut chercher dans certaines de leurs formes le point de départ de civilisations ultérieures. Fait remarquable, à l'opposé des résultats de l'analyse des sédiments et des études de la flore, prèsque tout caractère spécifique propre aux époques de chaleur et de froid fait défaut dans l'image des restes de mammifères que nous possédons.

S u m m a r y. From 1954-1956 in the Bockstein area (Lonetal, Württemberg) the layers of the Brandplatte and of the Hang on the lower side of the Schmiede were excavated and as far as was technically possible - were traced down to the deposits of the valley bed. In both the recent and earlier excavations analytical tests for sediments, fauna and flora were undertaken (Elizabeth Schmid, Ulrich LemmanN, Paul Filzer). Judging from the results which have sofar come to hand, the violet layer of the Bocksteinloch with its Hand-AxeCulture must have been formed in a markedly warm phase of the Riss-Würm-Interglacial (using these terms in the classical sense), while the somwhat older layer of the main culture of Hand-Axes of the Bocksteinschmiede points to an earlier and colder period of the same warm phase, or to rather late stage of the outgoing Riss-Glaciation. Older still than the main culture of the Schmiede is a great pile of layers which presents once again a varied interchange of sediments produced in warm and cold periods. The sequence of Hand-Axe-Cultures above all the main culture of the Schmiede - appears to be so old that in it certain germinal forms of later culture-development may be sought. In noticeable contrast with corresponding sedimental-analytical and floristical results of examination there is a complete gap in the records of mammal remains as far as their specific attribution to colder or warmer periodes is concerned. 
Mit ihrer Exkursion am 21. September 1953 gab mir die Stuttgarter Tagung der Deutschen Quartärvereinigung den Anstoß zu einer vorläufigen Außerung über Ergebnisse und Probleme der "Quartärforschung im Lonetal" (Eiszeitalter und Gegenwart 4/5, 1954). Die Datierung der seit 1932 insbesondere am Bockstein neu erschlossenen Kulturen konnte 1953/54 nur in Vermutungen erörtert werden. Es war zu hoffen, die Fortsetzung der Arbeiten werde bald die eine und andere, gewissere Antwort auf offene Fragen erlauben und damit endlich auch die ausführliche Veröffentlichung der Befunde ermöglichen. Es bedurfte aber noch dreier, jeweils sechswöchig mit verstärkter Belegschaft durchgeführter Grabungen, bis die drängendsten Probleme zwar immer noch keineswegs "gelöst" waren, Lösungen aber doch auf breiterer und festerer Grundlage erwogen werden konnten. Solche Erwägungen können heute zudem durch Erkenntnisse gestützt werden, die mittlerweile von den Spezialuntersuchern gewonnen wurden - von Elisabeth SCHmid (Schichten und Schneckenreste), Ulrich LeHmanN (Skelettreste von Wirbeltieren), Paul Filzer (Pflanzenreste), mit deren freundlicher Erlaubnis ich einige Ergebnisse ihrer, der ausführlichen Veröffentlichung zugehörigen Bearbeitungen hier vorweg nenne. Der kurze, folgende Bericht möge die wichtigsten Fragen der Datierung unserer Bocksteinkulturen in konzentrierter Form zur Diskussion stellen, während nach genau 25 Jahren meiner eigenen Beteiligung an den 95 Jahren urgeschichtlicher Lontalforschung - der erste Band des Bocksteinbuches bei Kohlhammer in Stuttgart gedruckt wird.

Am Abhang unterhalb der Bocksteinschmiede wurden in den Jahren 1954, 1955 und 1956 unerwartet mächtige und vielfältig gegliederte Schuttlagen in einem $7.50 \mathrm{~m}$ breiten Abbaustreifen bis auf den gewachsenen Fels des tertiär ausgelaugten Talskelettes abgetragen. Was 1953 in einem "Gräbele“ erst eben angeschnitten war, konnte seither in Profilen bị zu $8.00 \mathrm{~m}$ Mächtigkeit aufgeschlossen werden. Der Fels der Talwand fällt so steil, daß er nur bis rund $16.00 \mathrm{~m}$ abwärts vom Vermessungsnullpunkt verfolgt werden konnte - siehe die Skizzen des Medianprofils und des Sagittalprofils + 5.50 F. in Taf. II $^{1}$ ). Von da ab talwärts waren die Schuttschichten nicht mehr bis zum Felsgrund aufzuschließen. Zwar vermochte der Bagger des Herrn Maurermeisters Hans Graf in Oberstotzingen gegen Ende der Grabungen des Jahres 1956 das Sagittalprofil + 5.50 F. erheblich tiefer freizuschachten, als dies in der Handarbeit mit dem Pickele oder höchstens einmal dem groben Pickel zu erreichen war; schließlich aber mußte auch die Baggergrabung mit der begrenzten Reichweite des Greifarms und in der Rücksicht auf die Sicherheit für die beteiligten Männer ihr immer noch vorzeitiges Ende finden. Ausschließlich dem Bagger ist es zu danken, daß wir endlich im Oktober 1956, lang nach dem Abschluß der Grabungen, mit einigen Wiesenschlitzen unsere Hangprofile in den Talgrund, allerdings auch hier nicht bis in die erwünschten Tiefen hinunter, fortsetzen konnten. Unsere Kenntnis der Schichten am Bockstein wurde 1955 und 1956 durch weitere Ausgrabungen am „Törle“ der alten Bocksteinhöhle, sowie durch einige Schacht-

1) Schnitte senkrecht zum Berghang nennen wir nach anatomischer Weise „sagittal“, Schnitte quer dem Hang entlang "frontal“. Bei der Anlage einer Grabung wird ein Sagittalschnitt womöglich so abgesteckt, daß er als "Mediane" ungefähr durch die Mitte der auszugrabenden Höhle und ihres Vorplatzes führt und weiterhin als Hauptbezugsebene für Abbau und Vermessung dienen kann. Was bergwärts liegt von einem, in der Mediane festgelegten, absoluten Nullpunkt, erhält die Bezeichnung + x.xx m S. (sagittal), was talwärts liegt, $-\mathrm{x}$ xx S.; was für den Blick vom Tal zum Berg links ist von der Mediane, + x.xx F. (frontal), was rechts ist, - X.xx F.; was über der Horizontale des absoluten Nuttpunkts liegt, + X.xx (Meterzahl in lateinischer Ziffer), was tiefer liegt, - X.xx. So heißt z. B. "Sagittalprofil + 5.50 F." die Ebene, die mit $5.50 \mathrm{~m}$ Zwischenraum links von der Mediane verläuft, oder "Frontalprofil + 3.60 S." die Ebene, die in $3.60 \mathrm{~m}$ Abstand bergwärts vom Nullpunkt die Sagittalen rechtwinklig schneidet. Mit „+ 5.50 F. / - 19.00 S. / - XII. 50" ist ein Punkt bezeichnet, der in der Sagittalebene $+5.50 \mathrm{~F}$. um $19.00 \mathrm{~m}$ talwärts von Null und um $12.50 \mathrm{~m}$ tiefer liegt als die Null-Horizontalebene. 
grabungen am Waldrand und in der Wiese unterhalb der alten Höhle erweitert; wir waren aber bisher nicht in der Lage, die Profile des „alten“ und des „neuen“ Bocksteins in durchgehenden Aufschlüssen so unmittelbar miteinander zu verbinden, wie dies als die via regia jeder Schichtenvergleichung immer anzustreben ist.

In der alten, stichwortmäßigen Formulierung „Riß oder Würm“ fragen wir vordringlich nach der zeitlichen Eingliederung der Faustkeilkultur, die mit der überwiegenden Menge ihrer mehr als tausend guten Stücke aus dem engeren Raum der eigentlichen „Bocksteinschmiede“ geborgen wurde und mit der Art ihres Inventars zu diesem Namen den Anlaß gab. Mit der sorgfältigen Ausarbeitung ihrer vielen schönen Werkzeuge, mit deren ungewöhnlichem Formenreichtum im Rahmen einer stilistisch gleichwohl streng umschriebenen Eigenart, findet die Hauptkultur der Schmiede vorzugsweise unser Interesse; die ganze Sippe etwas jüngerer und auch älterer Bocksteinkulturen wird, der Hauptkultur mehr oder minder ähnlich, am besten von ihr aus vergleichend morphologisch betrachtet. Wie wir aber die frühen Bocksteinkulturen schließlich in ihrer Bedeutung für die europäische und gesamtmenschliche Urgeschichte zu bewerten haben, hängt entscheidend von der Altersbestimmung $a b$, die der gleichen Kultur, je nachdem, den Charakter eines wegeweisenden Vorläufers anderwärts vertretener Kulturen, ihres Zeitgenossen, oder auch des zurückgebliebenen Spätlings verleihen kann. Als ein solcher Nachkömmling westeuropäischer Vorbilder müßte unsere Schmiedenkultur gelten, wenn sie wirklich in den unteren Lagen eines darüber durchweg „kalten“ Schichtenstoßes aus der letzten großen Vereisungszeit gelegen wäre. RIEK postuliert eine solche stratigraphische Lage für sein „Jung-Acheuléen“ (Die Eiszeitjägerstation am Vogelherd I. 1934, S. 100), dessen einziger, kleiner Faustkeil (Taf. V. Fig. 3) zur Hauptkultur der Schmiede durchaus passen könnte. Seitdem ich - nach anfänglicher Übereinstimmung - an der Richtigkeit der RIEK'schen Deutung des Vogelherdprofils (S. 37 bis 39) zweifle und mindestens ihre naheliegende Ubertragung auf die Bocksteinschmiede ablehne, muß ich mich sichern gegen den Einwand, ich wünschte mehr, als daß ich es beweisen könnte, meiner Hauptkultur der Bocksteinschmiede sei mit einem höheren erdgeschichtlichen Alter auch der höhere kulturelle Rang zuzuerkennen. Tatsächlich ließ mich zuerst die eindrücklich ursprüngliche Schönheit der Schmiedenkultur vermuten, sie sei älter als früh-würmeiszeitlich; aber wenn ich weiterhin auch alle stratigraphischen Anhalte für die Möglichkeit eines höheren Alters sorgsam beachte, so mußte doch die Frage der Datierung unserer alten Bocksteinkulturen zunächst ausdrücklich offen bleiben (1954, S. 128-131).

So war ich auch am Morgen des 1. September 1954 beim Frühstück im Bocksteinhäusle mit Paul Woldsted und Elisabeth SCHMid darüber einig gewesen, daß meine Vermutung eines wärmezeitlichen Hiatus in der Schichtenfolge über der Schmiedenkultur vielleicht richtig, vorerst aber unbewiesen sei, und daß wir so lange im Zweifelsfall bei der würmeiszeitlich „kalten“ Deutung des ganzen Profiles bleiben müßten. Kaum war nach dieser Diskussion Elisabeth SCHMID vom Bockstein abgereist, als Paul Filzer ihn bestieg und uns mit der ganz nebenbei gemurmelten Mitteilung alarmierte, er habe einige Kohlestückchen aus der violetten Schicht des Bocksteinlochs (Taf. II A; 1954 Medianschnitt und Abb. 21 G/f) als Eichen- (Quercus), Ahorn- (Acer), Hagbuchen- (Carpinus) und (wahrscheinlich) Haselholz (Corylus) bestimmt. Die Kohlen waren am 4. 9. 1934 auf der Fläche +11.00 bis $+13.00 \mathrm{~S} . / \pm 0$ bis $-2.00 \mathrm{~F}$. des Bocksteinlochs in der violetten Schicht gefunden worden; mit vielen anderen Klein- und Feinfunden waren sie „verloren“, nachdem der Erbe meines Tübinger anatomischen Lehrstuhls meine Sammlungen aus dem Institut evakuieren ließ, während ich in Gefangenschaft abwesend war. Erst 1954 hatte ich durch Zufall die Kohlestückchen, zum Glück noch wohlverpackt, mit aller originalen Herkunftsbezeichnung wiedergefunden, und ohne einen Gedanken daran, daß gerade sie einen Laubwald dokumentieren könnten, 
der Ordnung und Vollständigkeit halber zur Bestimmung an FILzER gegeben. Sein unerwarteter Untersuchungsbefund brachte den bisher vermißten Nachweis dafür, daß die violette Schicht, über der Hauptkultur gelegen und jünger als sie, auf begrenzter Fläche im Schutz der Höhle erhalten, auch reell jene Wärmezeit repräsentiert, die ich bisher nur im virtuellen Hiatus - und, mit der Oberfläche des gelben Lehms, an falscher Stelle - vermutet hatte. Die Nachuntersuchung einer noch vorhandenen Erdprobe aus der, seit 1935 ganz abgebauten, violetten Kulturschicht ergab in vereinzelten Pollen eine Bestätigung, zwar nicht für den leicht verderblichen Ahorn (Acer), wohl aber für Eiche (Quercus), Hagbuche (Carpinus) und jetzt auch sicher Hasel (Corylus). Dazu kam Weide (Salix) und vor allem Kiefer (Pinus), außerdem sind Korbblütler (Compositae), Doldengewächse (Umbelliferae), Gräser (Gramineae) und (vielleicht) Wegerich (Plantago?) gefunden worden. Die immer schwebende Frage, wie weit der Bestand an Holzkohlen durch kulturelle Auslese bedingt sein könnte, ist hier bemerkenswerter Weise im Sinne einer bevorzugten Laubholzfeuerung beantwortet. Keinesfalls aber vermag ein pollendiagnostisch erwiesenes Vorkommen der Kiefer neben den Laubhölzern das wärmezeitliche Bild der Baumflora zu verwischen.

Der Wärmewald der violetten Schicht bewegte uns umso mehr, als wir wenige Wochen vorher eine weitere, eindeutig wärmezeitliche Ablagerung kennen gelernt hatten. Im großen Graben, dem 1953 erst angefangenen Sagittalschlitz entlang der Mediane, war am 9. 8. 1954 die schwarzviolette Schicht angeschnitten und seither streckenweise abgebaut worden. Zum großen Teil aus locker gepacktem, grobem bis gröbstem Kalkschutt bestehend, mußte diese auffallende Schicht mit dem erheblichen Humusgehalt ihrer lose kleinsteinigen Lehmfüllung, mit einer Unmenge wohlerhaltener Gehäuse von Waldschnecken und mit kleinsten Stücken von überwiegend Laubholzkohlen, schon für die oberflächliche erste Betrachtung als wärmezeitlich erscheinen. Die Befürworter eines würmzeitlichen Alters unserer Faustkeilkulturen, insbesondere der Hauptkultur der Schmiede, sahen denn auch unbedenklich in der schwarzvioletten Schicht einen Niederschlag der Riß/Würm-Interglazialzeit im klassischen Sinne dieser Bezeichnung. Jetzt, nach dem Nachweis eines Wärmewaldes in der violetten Schicht, mußte neu mit allen Mitteln nach einer gültigen Deutung der Schichtenfolge gesucht werden. So grundsätzlich methodisch wir dabei von der Priorität der unmittelbaren oder, bei getrennten Profilen, der „accordweisen“ Schichtenvergleichung ausgehen (WETZEL 1954, S. $113 \mathrm{ff}$ ) und jeder typologischen Parallelisierung die Auswertung aller stratigraphischen, sedimentanalytischen, faunistischen und floristischen Differenzierungsmöglichkeiten vorziehen, so ist doch auch die hilfsweise Beiziehung der Kulturvergleichung in bestimmten Grenzen und unter bestimmten Umständen erlaubt, ja geboten und unentbehrlich. Die vergleichsweise Verwertung widerspruchslos festgestellter, großer Züge der kulturellen Entfaltung („geschlossene Faustkeilkulturen sind älter als einheitliche Klingenkulturen mit Knochenwerkzeugen") ist der Urgeschichte so gut erlaubt, wie der Paläontologie die Anwendung des - für alle erst zu klärenden Einzelfragen untauglichen - Leitfossilprinzips im Rahmen klarer großer Linien der erdgeschichtlichen Entfaltung der Organismenwelt; die Nächstvergleichung der Aufschlüsse unserer „Profilnester“ am Bockstein erlaubt darüber hinaus eine Ausdeutung manches typologischen Befundes, die der Fernvergleichung versagt werden muß.

In den ausgewählten Ausschnitten bezeichnender Profile sollen mit der Tafel II die stratigraphischen Vergleichungsmöglichkeiten der am "neuen“ Bockstein 1932 bis 1956 aufgeschlossenen Schichten sichtbar gemacht werden. Mit den Verbindungslinien zwischen den Schichtengrenzen verschiedener Profile sind die Lageverhältnisse bezeichnet, auch für Ablagerungen, die sich nirgends unmittelbar im selben Profil berühren, sich durch kettenweise Verzahnungen aber doch zueinander ordnen lassen; besonders deutlich wird das vielfältige Auskeilen der Schichten mit den entsprechenden Hiatus. So kann der 
Entwurf zu einem Idealbild der wichtigsten Ablagerungen aus dem Profilvergleichungsschema unmittelbar abgelesen werden. Hier sei zunächst für die Schmiede mit dem Loch, die Brandplatte und den Abhang eine solche „ideale“ Schichtenfolge zusammengestellt und mit kurzen Angaben über bereits vorliegende Ergebnisse der sedimentanalytischen, faunistischen und floristischen Spezialuntersuchungen verbunden. Für jede einzelne Schicht ist angegeben, in welchen Profilen der Tafel II sie vertreten ist.

$\mathrm{Humus}-\mathrm{Kalkschutt}$ (B.C.D.E.F.G.). Oben grobsteiniger, in tieferen Lagen fein- bis feinststeiniger Kalkschutt mit schwarzer Humuserde. Spärliche Reste einer nach-eiszeitlichen Wildfauna.

B r a u n e K r u me (H.J.K.L.). So gut wie steinlose, grau- bis rötlichbraune, lockere (lössig erscheinende) Erde.

Die beiderlei Oberflächenschichten sind homolog; im Medianprofil bei -28.00 S., im Sagittalprofil +5.50 F. bei -33.00 S. löst die braune Krume den Humuskalkschutt ab. Beide Oberflächenschichten können als nacheiszeitalterliche Bildung gelten.

B l a nke r Bergkies (A.B.C.D...F.). Kleinsteiniger Kalkschutt mit wenig, stellenweise ohne Lehmfüllung. UUbergang von kälterem zu wärmerem Klima. - Wolf (Canis lupus), Höhlenbär (Ursus spelaeus), Dachs (Meles meles), Kleines Wildpferd (Equus przewalski), Ren (Rangifer). - In oberen Lagen Eiche (Quercus), Ahorn (Acer).

O be r e r L öß (A...C.). Graugrünlicher Löß mit stellenweise groben Kalkbrocken. Trokkene Kältezeit. - Panther (Panthera spelaea, Höhlenlöwe), kleines Wildpferd (Equus przewalski), Wollnashorn (Coelodonta antiquitatis), Ren (Rangifer). - Kiefer (Pinus), Korbblütler (Compositae), Farne (Filices).

Fe in ste in iger Lehm (A.B.C.D.). Gelblich-bräunlicher Lehm mit feinem Kalkschutt. - Trocken-kühles Klima einer "Wärmeschwankung“. - Feldmaus (Microtus agrestis arvalis), Hyäne (Hyaena spelaea, Braunbär (Ursus arctos), kleines Wildpferd (Egnus przewalski), Wollnashorn (Coelodonta antiquitatis), Ren (Rangifer), Wildrind (Bos sp. ind. - Schnecke Ariantha.

L e h mige r B e rgkies (E.F.G.). Brauner Lehm mit feinem Kalkschutt, dem feinsteinigen Lehm homolog, mit ihm durch Frontalprofile zwischen der Mediane und dem Sagittalprofil $+5.50 \mathrm{~F}$. unmittelbar verbunden.

$\mathrm{Streifiger}$ gelber Lehm (A...C.D.), talwärts zunehmend steinig und nicht mehr streifig (E.F.G.). Kältezeitlich abgelagertes, in einer Auftauphase durchnäßtes und verschwemmtes Sediment. - Hase (Lepus), Erdmaus (Microtus agrestis), Wolf (Canis lupus), Rotfuchs Vulpes vulpes), Wiesel (Mustela nivalis), Hyäne (Hyaena spelaea), Panther (Panthera spelaea), Mammut (Elephas primigenita), großes Wildpferd (Equus germanicus), Wollnashorn (Cododonta antiquitatis), Ren (Rangifer), Rothirsch (Cervus elaphus), Wildrind (Bos sp. ind.).

B rauner Grobschutt (B.C.). Mittelgrober Kalkschutt in gelblich-bräunlichem Lehm. Ablagerung einer Kaltzeit. - Murmeltier Marmotta marmotta), Wolf (Canis lupus), Rotfuchs (Vulpes vulpes), Höhlenbär (Ursus spelaeus), Hyäne (Hyaena spelaea), Panther (Panthera spelaea), Mammut (Elephas primigenius), großes Wildpferd (Equus germanicus), Wollnashorn (Coelodonta antiquitatis), Ren(Rangifer), Hirsch (Cervus sp. ind.), Wildrind (Bos sp. ind.), Moschusochse (Ovibos moschatus).

Der braune Grobschutt liegt eindeutig unter dem gelben Lehm (C.) und über der Hauptkulturschicht (B.); er hört aber nach dem Höhlenhintergrunde zu bei $\nmid 7.80 \mathrm{~S}$. des Medianprofils auf (1954, Abb. 5), überschneidet sich also nirgends mit der hellbraunen und vor allem der violetten Kulturschicht des Bocksteinlochs, die erst nach $+8.50 \mathrm{~S}$. beginnen. So mußte es der ScHMID'schen Sedimentuntersuchung vorbehalten bleiben, über die stratigraphisch offene Eingliederung dieser Schicht $\mathrm{zu}$ entscheiden. Es zeigte sich, daß der braune Grobschutt (von mir früher als „brauner Schotter" bezeichnet) zum hangenden gelben Lehm in nächster' Beziehung steht, daß er also unmittelbar unter ihm und über der violetten Schicht in die Schichtenfolge einzugliedern ist. Nachdem ich im Anblick des Profils geglaubt hatte, eine braune Lehmschicht dicht über der Hauptkultur bei +0.30 bis +2.70 entlang der Mediane dem höhlenwärts anschließenden braunen Grobschutt zurechnen zu dürfen, ließ ich mich in dieser Annahme durch Elisabeth ScHмID dahin berichtigen, daß dieser Schichtbereich dem feinsteinigen Lehm zuzurechnen sei.

F e in k i e s (C.). Kleinststeiniger Kalkschutt, fast ohne Lehm dazwischen, als "Querschachtskies“ im begrenzten Bereich um + 2.50 F./3.50 S. aufgeschlossen. Äußerlich ähnlich dem oberen, „kalten“ bis „kühlen“ Bergkies. - Hamster (Cricetus cricetus), Wolf (Canis lupus), Rotfuchs (Vulpes vulpes), Höhlenbär (Ursus spelaeus), Dachs (Meles meles), Hyäne (Hyaena spelaea), großes Wildpferd (Equus germanicus), Wollnashorn (Coelodonta antiquitatis), Ren (Rangifer), 
Rothirsch (Cervus elaphus), Hirsch (Cervus sp. ind.), Wildrind (Bos sp. ind.), Auerhuhn (Tetrao urogallus).

Wie der braune Grobschutt, so steht auch dieser untere Feinkies in keiner unmittelbaren Lagebeziehung zur violetten Schicht. Eine sedimentanalytische Qualifizierung liegt noch nicht vor; ich stelle die Schicht unter Vorbehalt dicht unter die "kalten“, hangenden Schichten des braunen Grobschutts und des gelben Lehms.

Violette Kulturschicht (A). Violettbräunlicher, „knollenmergelfarbiger“ Lehm mit mäßig vielen, mittelgroben Kalkstücken. Warm-trocken-kontinentales Ablagerungsklima. Wolf (Canis lupus), Rotfuchs (Vulpes vulpes), kleiner Fuchs (Alopex lagopus?), Höhlenbär (Ursus spelaeus), Dachs (Meles meles) Hyäne (Hyaena spelaea), großes Wildpferd (Equus germanicus), Wollnashorn (Coelodonta antiquitatis), Ren (Rangifer), Rothirsch (Cervus elaphus), Hirsch (Cervus sp. ind.), Wildrind (Bos sp. ind.), Auerhuhn (Tetrao urogallus). - Eiche (Quercus), Ahorn (Acer), Hagbuche (Carpinus), Hasel (Corylus), Weide (Salix), Kiefer (Pinus), Doldengewächse (Umbelliferae), Korbblütler (Compositae) Gräser (Gramineae), vielleicht Wegerich (Plantago?).

$\mathrm{Hellbraune} \mathrm{Kulturschicht}$ (A.). Hellbraun-locker-lehmige Schicht mit wenig feinem Kalkbruch. - Hase (Lepus), Wolf (Canis lupus), Dachs (Meles meles), großes Wildpferd (Equus germanicus), Hirsch (Cervus sp. ind.), Wildrind (Bos sp. ind.)

Ti e f e r e r L öß (E.). Fast steinloser - allerdings durch eine Kalkschuttlage unterbrochener - Löß, offensichtlich verschwemmt, also vielleicht primär noch älter, als es seiner sekundär endgültigen Lagerung entspräche, zwischen der "roten Faustkeilzone" des Hangs und dem steinigen Lehm - als ein begrenzter Keil von -7.60 bis rund $-14.00 \mathrm{~S}$. am Felsabfall entlang der Mediane. Ein teilweises Übergreifen oberster Teile der Faustkeilschicht nicht nur zwischen, sondern auch über den tieferen Löß läßt vermuten, daß er gegen Ende der Faustkeilzeit abgelagert oder verlagert wurde. Die eingehende Erörterung der verwickelten Stratigraphie und noch verwickelterer Deutungsmöglichkeiten kann erst nach dem Vorliegen der Sedimentanalyse weitergeführt werden. Stratigraphisch kann eine Abschwemmung des tieferen Lösses nicht der Lehmverschwemmungsphase zugerechnet werden, die Elisabeth Schm:D für die Zeit nach der Ablagerung des gelben Lehms ansetzt. Sie müßte vielmehr in einer Naßzeit am Ende „einer der Faustkeilzeiten" erfolgt sein. „Einer" ..., weil bisher nicht auszumachen ist, welcher der Faustkeilschichten bei der Schmiede und im Loch die rote Faustkeilzone des Hangs entspricht.

$\mathrm{Hauptkulturschicht}$ der Schmiede (A.B.C.). Sepiabraune Lehmerde mit kleinstückigem Kalkschutt. Feucht-gemäßigtes Ablagerungsklima. - Hase (Lepus), Murmeltier (Marmotta marmotta), Wolf (Canis lupus), Rotfuchs (Vulpes vulpes), Alpenwolf (Cuon alpinus), Höhlenbär (Ursus spelaeus), Panther (Panthera spelaea), Mammut Elephas primigenius), großes Wildpferd (Equus germanicus), Wollnashorn (Coelodonta antiquitatis), Ren (Rangifer), Rothirsch (Cervus elaphus), Riesenhirsch (Megaceros giganteus), Auerochse (Bos primigenius), Gemse (Rupicapra rupicapra). - Hasel (Corylus), Kiefer (Pinus).

Braune bis orangebraune Kultur an der Brandplatte (D.). Bräunlichrötlichgelber Lehm mit feinem Kalkbruch, in unmittelbarer Fortsetzung der Hauptkulturschicht der Schmiede. - Wolf Canis lupus), Rotfuchs (Vulpes vulpes), großes Wildpferd (Equns germanicus), Auerhuhn (Tetrao urogallus). - Eiche (Quercus).

Rote Faustkeilkultur am Hang (E.G.). Lockere Erde mit mittelgrobem bis feinem Kalkbruch, rotbraun, nicht "violett-rot", am Hang vor allem im Bereich des großen Grabens vertreten, nur mit einer Zunge bei -19.00 bis -24.00 S. in das Sagittalprofil + 5.50 F. hinüberreichend. Unmittelbarer Schichtenzusammenhang mit der Faustkeilzone der Brandplatte im Bereich des Sagittalprofils $+2.50 \mathrm{~F}$., damit auch mit der Hauptkulturschicht. Hase (Lepus), Lemming (Lemmus sp. ind.), Wolf (Canis lupus), Fuchs Vulpes sp. ind.), Dachs (Meles meles), Wiesel (Mustela nivalis), Panther (Panthera spelaea), Hyäne (Hyaena spelaea), Wollnashorn (Coelodonta antiquitatis), großes Wildpferd (Equus germanicus), Ren (Rangifer), Rothirsch (Cervus elaphus), Birkhuhn (Lysurus tetrix).

Dunkler Grobschutt und oberer Brand (A.B.C.). Grober Kalkschutt mit dunkler bis kohlenschwarzer Lehmerde, in der eigentlichen Brandschicht geröstete Kalksteine. Feucht-gemäßigtes Klima, wie zur Zeit der Hauptkultur. - Wühlratte (Arvicola terrestris, Wolf (Canis lupus), Rotfuchs (Vulpes vulpes), Höhlenbär (Ursus spelaeus), Dachs (Meles meles), Hyäne (Hyaena spelaea), Panther (Panthera spelaea), großes Wildpferd (Equus germanicus), Rothirsch (Cervus elaphus), Wildrind (Bos sp. ind.). - Kiefer (Pinus), Fichte (Picea).

$\mathrm{Fe}$ in e bra une Erde in der Schmiede (B.). Feine, lockere braune Lehmerde fast ohne Steine. Kühl, trocken. - Maulwurf (Talpa europaea), Wühlratte (Arvicola terrestris), Feldmaus (Microtus agrestis arvalis), Rotfuchs (Vulpes vulpes), Hyäne (Hyaena spelaea), großes Wildpferd (Equus germanicus), Wollnashorn (Coelodonta antiquitutis), Riesenhirsch (Megaceros giganteus). 
Feine gelbe Erde in der Schmiede (B.). Feine, lockere gelbliche Lehmerde mit feinstem Kalkbruch. Kühl, trocken. - Wühlratte (Arvicola terrestris), Rotfuchs (Vulpes vulpes), Braunbär (Ursus arctos), Hyäne (Hyaena spelaea), großes Wildpferd (Equus germanicus), Wollnashorn (Coelodonta antiquitatis).

Feiner brauner Lehm der Brandplatte und des $\mathrm{Hangs}$ (C.D...F.G.). Lockere, gelblich braune Lehmerde mit grobem Kalkbruch, an der Basis der untere Brand. Noch ohne Sedimentanalyse. - Hase (Lepus), Maulwurf (Talpa europuea), Mausohr (Myotis myotis), Hamster (Cricetus cricetus), Wühlratte (Arvicola terrestris), Erdmaus (Mycrotus agrestis), Fuchs (Vulpes sp. ind.), Dachs (Meles meles), Panther (Panthera spelaea), Hyäne (Hyaena spelaea), großes Wildpferd (Equus germanicus), Rothirsch (Cervus elephus), Birkhuhn (Lysurus tetrix). Kiefer (Pinus), Fichte (Picea), Ulme (Ulmus), Eiche (Quercus), Weide (Salix), Farne (Filices).

Die genaue stratigraphische Parallelisierung des tiefen feinen braunen Lehms mit bestimmten, tiefen Lehmen der Schmiede und des Bocksteinlochs war in den Aufschlüssen nicht zu gewinnen. Die beiderlei Feinlehme der Schmiede sind ganz isolierte Schichtflecke in der Schmiedenvertiefung (1954, Medianprofil Abb. 5. +1.00 bis +2.60 S.), ihrerseits von einem ebenso isolierten tiefsten Feinlehm unterlagert. Er könnte, muß aber nicht dem braunen Feinlehm.... oder auch dem folgenden roten Tiefenlehm entsprechen.

$\mathrm{R}$ o te r T i e f e n lehm (A.B.). Zwischen großen Kalktrümmern liegender, rötlicher feiner Lehm, im Bocksteinloch. Nach sedimentanalytischer Beurteilung einer nassen Auftauphase entsprechend. - Wolf (Canis lupus), Rotfuchs (Vulpes vulpes), Hyäne (Hyaena spelaea), großes Wildpferd (Equus germanicus), Wollnashorn (Coelodonda antiquitatis), Riesenhirsch (Megaceros giganteus), Hirsch (Cervus sp. ind.), Wildrind (Bos sp. ind.).

Der rote Tiefenlehm, da und dort nur noch von eigentlichem „Bodenspeck“ unterlagert, ist bei anscheinend ununterbrochenem Zusammenhang mit den hangenden feinen Lehmen am ehesten als deren basaler Beginn anzusprechen. Ganz gesichert ist auch diese Vermutung nicht; vor allem kann weder behauptet noch ausgeschlossen werden, daß tiefste Feinlehmlagen mit gewissen Tiefenschichten des Hangs zusammengehören.

$\mathrm{RotbraunerFeinschutt}$ a m Hang (E.F.G.). Feiner Kalkschutt mit wenig rotbrauner, lockerer Lehmerde. Noch ohne Sedimentanalyse. Keine bestimmbaren Tier- und Pflanzenreste.

Der rotbraune Feinschutt liegt in seiner größten Mächtigkeit im Südostabschnitt des Abbaufeldes ohne zwischengeschalteten feinen Lehm dicht unter der roten Faustkeilschicht. Nach Nordwesten zu liegt er unter dem braunen Feinlehm und verliert dabei an Mächtigkeit wie an facieller Prägnanz.

Gelber S te in lehm (E.F.G.). Chromgelber Lehm mit viel mittelgrobem Kalkschutt. Noch ohne Sedimentanalyse. - Murmeltier (Marmotta marmotta), Fuchs (Vulpes sp. ind.), großes Wildpferd (Equus germanicus), Zwergtaucher (Podicipes fluviatilis), Amphibia. - Nadelhölzer (Coniferae). Der chromgelbe Steinlehm gehört, über die ganze Abhangabbaufläche vertreten, zu deren bezeichnendsten Sedimenten.

$\mathrm{V}$ i o l e t t e r F e in schut $\mathrm{t}$ (F.G.). Feinschutt mit ganz wenig, violettbrauner Lehmfüllung. Noch ohne Sedimentanalyse. Keine Tier- und Pflanzenreste. Die Schicht ist nur im Nordwestabschnitt des Hangs ab $-14.00 \mathrm{~S}$. talwärts vertreten.

Blanker Grobschutt (E.F.G.). Straßenschottergrober Kalkschutt ohne jede Lehmfüllung, ohne Tier- und Pflanzenreste, im ganzen Hangfeld ab $-14.00 / 13.00 \mathrm{~S}$. talwärts vorhanden.

$\mathrm{Schwarzviolette} \mathrm{Schicht} \mathrm{(E.F.G.).} \mathrm{Gröbster} \mathrm{Kalkbruch,} \mathrm{daneben} \mathrm{allerfeinster}$ Kalkschutt, mit schwarzvioletter, feiner lockerer Lehmerde. -- Reh (Capreolus capreolus), Kleinsäuger (Mammalia minima), Schnecken eines warmen, feuchten Waldes. - Hainbuche (Carpinus), Hasel (Corylus), Schwarzdorn (Crataegus), Kiefer (Pinus), Fichte (Picea), Beifuß (Artemisia). Auch die schwarzviolette Schicht ist, etwa ab -10.00 S. talwärts, am ganzen Hang vertreten.

$\mathrm{O} c k$ ergelbe Feinschutte (E.F.G.). Feinschuttlagen von wechselnden Korngrößen mit sehr wenig, kräftig ocker- bis orangegelber Lehmbeteiligung. - Fuchs (Vulpes sp. ind.), Kleinsäuger (Mammalia minima), Vögel (Aves). Am ganzen Steilabfall des Hangs liegt der ockergelbe Feinschutt dicht auf dem gewachsenen Fels. Sinterzone in der Schichtoberfläche von -10.00 bis $-14.00 \mathrm{~S}$. des Nordwestfeldes am Hang.

$\mathrm{Fe}$ in e r t i ef e $\mathrm{r}$ L e hm (F.). Gelblicher (lössiger?) Lehm mit wenig grobem Kalk. Bisher nur in kleiner Fläche im Sagittalprofil +5.50 F. am Fuß des Felsabfalls aufgeschlossen, unter den ockergelben Schutten. Der tiefe feine Lehm könnte - wenn größere und zusammenhängende Aufschlüsse zu erzielen wären - eine schlüsselmäßige Bedeurung gewinnen, sowohl für die bergwärtige Parallelisierung (tiefe feine Lehme an der Schmiede und im Loch), als auch für die Zusammenhänge mit den Talgrundsedimenten. 
Die Zusammenstellung zeigt, daß dem Entwurf eines Idealprofils der Bocksteinschichten auch heute noch große Schwierigkeiten und Lücken der Befunde gegenüberstehen. Viele unserer Schichten, die in den ersten Aufschlüssen nicht zueinander zu ordnen waren, konnten durch die zusätzlichen Grabungen der letzten Jahre in eine klare Folge eingegliedert werden; für wesentliche Zusammenhänge aber fehlt die unmittelbar stratigraphische Verzahnung. Auch die sedimentanalytischen, faunistischen und floristischen Sonderuntersuchungen zeigen einschneidende Lücken, die nur zum Teil auf technische Verzögerungen, im Bereich der Faunen und der Floren viel mehr auf materialbedingte Unzulänglichkeiten zurückzuführen sind.

Die Wirbeltierreste sind in allen Schichten des neuen Bocksteins erstaunlich wenig Lahlreich, in manchen kümmerlich oder überhaupt nicht vertreten. Sehr umstreitbar ist vielfach ihr Wert als Zeugen für bestimmte Klimate; denn wir wissen, daß höhere Wirbeltiere, Vögel und vor allem Säuger, in weitgehender Umstellungsfähigkeit ihre Lebensgewohnheiten in sehr viel kürzeren Zeiträumen ändern können, als sie seit „unseren“ eiszeitalterlichen Bocksteinzeiten vergangen sind. Überschneidungen bei Klimawechsel sind sowieso selbstverständlich anzunehmen. Wühlende Erdbewohner scheiden weitgehend aus der Faunenbegutachtung aus, weil sie viel später in die Schicht gekommen sein können. So könnte eigentlich erst die sorgfältige Feststellung kleinerer quantitativer Schwankungen in der Beteiligung von „eher kälteliebenden“ und „im Zweifelsfall mehr wärmeliebenden " Tieren gewisse klimatische Veränderungstendenzen erkennen lassen. Gerade dafür aber ist unser, überwiegend kulturell ausgelesenes Material an Wirbeltierresten ganz und gar ungenügend. Je „niederer" die Tiere, umso geringer ist, allgemein, ihre biologische Plastizität, und umso eher können klimagebundene Formen vorkommen. Die Schnecken wären, in uralten Schichten oft "wie neu" erhalten, mit vielen Arten in diesem Sinne brauchbar - aber sie sind in nur ganz wenigen Schichten genügend vertreten. Dazuhin sind viele der 1932-35 sorgsam gesammelten Schnecken seit der Zwangsverlagerung meiner Sammlungen verschwunden.

Am sichersten „leiten“ klimatisch bestimmte Pflanzengruppen, vorzüglich die besonders gut erhaltenen Baumpollen. Zwar kann in unseren Bocksteinschichten von einer regelrechten quantitativen Pollenanalyse keine Rede sein. In vielen Schichten sind überhaupt keine, in anderen nur vereinzelte Pollen vorhanden bzw. erhalten, und so konnten wir in den Untersuchungen, die, 1932 mit noch sehr unzureichenden Mitteln begonnen, erst seit 1953 durch FILzen unter Ausnützung aller seither entwickelten Möglichkeiten durchgeführt werden, nur eben das Vorkommen bestimmter Formen registrieren, aber weder - bis auf den günstigsten Ausnahmefall - ihr gegenseitiges Mengenverhältnis bestimmen, noch dessen Veränderungen von Schicht zu Schicht jemals durchgehend verfolgen. Immerhin kann das Vorhandensein bestimmter Baumarten, und sei es nur durch jeweils ein einziges Pollenkorn belegt, maßgebend bezeichnend sein für klimatische Verhältnisse, ohne die das Wachsen auch nur eines einzigen Baumes solcher Arrt, geschweige denn etwa einer ganzen Laubholzgruppe, unmöglich gewesen wäre. Die Pollenfunde werden in manchen Schichten durch zerstreute oder in Brandlagen gehäuftere Holzkohlestückchen ergänzt. Sehr viel mehr zwar, als die Pollen, unterliegen die Kohlen der kulturellen Auslese; gerade die Kohlen aber sind meistens genau zu bestimmen und sowieso als Baumwuchszeugen besonders wertvoll für klimatische Hinweise.

Suchen wir so, unter vorzugsweiser Auswertung der Sedimentbeschaffenheit und der Pflanzenreste mit reservierter Beiziehung der faunistischen Befunde, nach Anhalten für eine klimatische Gliederung unserer Bocksteinschichten und ihre Einordnung in das klassisch geläufige Schema der Kälte- und Wärmezeiten des Eiszeitalters, so gilt unsere erste Frage nach wie vor der violetten Faustkeilkultur des Bocksteinlochs. Sedimentanalyse und Flora weisen einhellig auf „warm“. Im Bild der Fauna „stört" dabei der 
kleine Fuchs, der als „Eisfuchs" läuft, von dem aber auch Lenmans betont, daß damit eine unbewiesene aktualistische Schlußfolgerung gezogen wird; wir wissen nicht, ob „kleine“ Füchse damit unbedingt kälteliebende Füchse waren. Wir können diese Überlegung nicht nur auf das Ren übertragen, das ja in so vielen Schichten mit dem Rothirsch zusammen gefunden wurde, sondern sogar auf das Wollnashorn, das fast in allen Schichten unseres „idealen“ Bocksteinprofils vorkommt und in keiner einzigen, erscheine sie sonst noch so eindeutig „warm“, durch das MERCK'sche Nashorn ersetzt ist. Abgesehen von der alten prinzipiellen Frage nach der Fraglichkeit alles "Leitens“, darf der Zweifel an der Klimabindung des Wollnashorns sogar auf seine „Wolle“ ausgedehnt werden. Wissen können wir von ihr nur, wo sie uns aus spätesten Phasen des Eiszeitalters in Natur oder im Bild überliefert ist; ob mit der bestimmten Zahnform immer, auch in früheren Eiszeitalterzeiten, die "Wolle" und damit die Kältebindung verknüpft war, müßte sich erst erweisen. Unsere eigenen Befunde brauchen ja nicht nur am Prokrustesbett früherer Schemata gemessen zu werden - sie sind auch selber neuer Befund und neues Ergebnis. Wenn in der violetten Kultur das „Woll“-Nashorn vorkommt, so müssen wir zur Kenntnis nehmen, daß dieses Tier auch in warmen LaubNadelholz-Mischwäldern noch leben konnte.

Es genüge für diese Mitteilung, das Problem der faunistischen Klimabindung oder Klima-Indifferenz am Beispiel der violetten Kultur genannt zu haben; es gilt für andere der kurz beschriebenen Schichten ebenso. Wie es auch mit ihrer Fauna sei, die violette Schicht bleibt „warm“. Die Frage ist, welche Wärmezeit sie repräsentiert. Noch immer könnte sie als die Wärmeschwankung zwischen dem ersten und einem zweiten Würmvereisungsvorstoß gelten, und es bliebe dabei, daß erst die schwarzviolette Schicht als das eigentliche Riß/Würm-Interglazial im klassischen Sinne anzusprechen wäre. Gegen diese Deutung spricht nicht einmal unbedingt das Bild der Baumflora. Auch im Würm-I/II-Interstadial kommen Laubholzarten vor, wie sie u. a. BRANDTNER (Archaeologia Austriaca V 1950, S. 104) für die "Göttweiger Verlehmungszone“ anführt. Der Eindruck des Botanikers Filzer, er habe es in der Flora der violetten Schicht mit einer vollgültig warmen Interglazialzeit zu tun und nicht nur mit einer interstadialen Schwankung, bedarf noch anderweitiger Stützung und Bestätigung.

Sie ergibt sich zunächst aus der Vergleichung mit den hangenden und den liegenden Gliedern des Profils. Nach Elisabeth Scнмm ist die warme violette Schicht nach oben gefolgt von einer niederschlagsreichen Vorstoßphase der I. Würmvereisungszeit, vertreten durch den kaltzeitlich abgelagerten braunen Grobschutt; der anschließende gelbe Lehm ist wohl ebenfalls noch kaltzeitlich entstanden, aber in einer folgenden Auftauphase durchnäßt und vielleicht verschwemmt, und er leitet damit über zu einer deutlichen Wärmeschwankung, die im kleinsteinigen Lehm ihren Niederschlag gefunden hat. Der darüber folgende Löß entspricht einer neuen II. Würmvereisungszeit, die mit dem blanken Bergkies ausklingt. Damit wäre „Göttweig“ oder „Fellabrunn“ (BrandtneR Eisz. u. Geg. 1954), das Würm-I/II-Interstadial, sogar wirklich in unserem Profil vertreten, so deutlich, wie wir dies gerne zur Kenntnis nehmen, ohne jemals „fordern“ zu dürfen, daß unsere Profile, deren beste und meistgliederige doch immer noch zum vielfach überwiegenden Teil aus Hiatūs bestehen, in irgend einer Hinsicht „vollständig“ seien. Dies gelte hier auch von vornherein gegenüber der immer noch denkbaren These, der kleinsteinige Lehm sei in Wirklichkeit ein "Paudorf“, also ein Würm-II/III-Interstadial, und es bleibe damit bei der alten Ansicht, die violette Schicht sei die WürmZwischenschwankung, die schwarzviolette Schicht das eigentliche Interglazial.

Dieser These aber widerspricht vor allem auch das Profil der liegenden Schichten. Schon die nächst tiefere, hellbraune Kulturschicht gehört offensichtlich nahe zur violetten Schicht und verleiht ihr eine gründlichere Basierung, als sie nur eben einer „Schwankung" zukommen sollte. Insbesondere aber scheint nach dem sedimentanalytischen Be- 
fund auch die Hauptkulturschicht der Schmiede nicht, wie ich selbst es zunächst getan hatte, einfach nach dem Schema „Mammut = Kältezeit“ zu beurteilen. Vielmehr schreibt ihr Elisabeth ScHMID samt dem darunter liegenden dunklen Grobschutt mit dem oberen Brand ein feucht-gemäßigtes Entstehungsklima zu, dem die sehr spärlichen Pflanzenreste wenigstens nicht unbedingt widersprechen. Erscheinen die darunter folgenden, feinen Lehme der Schmiede zu kühlerer und trockenerer Zeit entstanden, so hat sowohl der rote Tiefenlehm der Schmiede und des Bocksteinloches, als auch vor allem der braune Feinlehm der Brandplatte, dort dicht unter der Faustkeilzone folgend, einem wieder ausgeprägt warmen Klima seine Entstehung zu danken, soweit wir dies aus den gut und zahlreich erhaltenen Pflanzenresten auch der zugehörigen unteren Brandlage schließen dürfen. So haben wir es in der violetten Schicht gar nicht mit einer vereinzelten Warmzeitablagerung zu tun, sondern mit dem jüngsten Glied einer ganzen Folge von wahrscheinlich zusammengehörigen Ablagerungen einer im Ganzen gemäßigten bis warmen, im Einzelnen rasch und mehrfach schwankenden Klimaperiode. Ob wir sie als dieses Ganze einheitlich nehmen und in ihr die Riß/Würm-Wärmezeit sehen wollen, oder ob ihre kühleren Phasen, vor allem in der Hauptkulturschicht ausgeprägt, noch als letzte Ausschläge der Riß-Kältezeit gelten sollen, mag vorerst offen bleiben. In keinem Fall dürften wir in einem solchen, ganzen "Warmschichtenkomplex" nur eine interstadiale $\mathrm{Zwischenphase} \mathrm{sehen.}$

Tief erst unter dem braunen Feinlehm als dem ältesten Glied dieses Komplexes folgt die schwarzviolette Schicht als die nächst ältere, eindeutig warmzeitlich entstandene Ablagerung. Von ihrem nächst jüngeren Nachfolger, jenem braunen Feinlehm, ist sie durch mehrgliedrige Schichtenlager von Mächtigkeiten bis über 2, ja fast $3 \mathrm{~m}$ geschieden. Die Spezialuntersuchung dieser Zwischenschichten steht noch aus; wir werden uns aber schwerlich getäuscht sehen in der vorgreifenden Vermutung, es seien im wesentlichen kältezeitlich entstandene Sedimente. Mit ihnen wären wir, wenn unsere schlüsselmäßig entscheidende Zuordnung der oberen Wärmeschichten richtig ist, schon mitten in der großen Riß-Eiszeit. In ihr (Riß-I/II), wenn nicht gar vor ihr (Mindel/Riß) liegt die schwarzviolette Schicht. Sehr alt, vielleicht uralt folgen darunter die vielfachen Lagen der ockergelben Feinschutte, die wohl wieder einer Kaltzeit entsprechen. Sie scheint mit der Durchnässung ihrer oberflächlichen Lagen, wie sie in deren streckenweiser Versinterung zum Ausdruck kommt, folgerichtig abgeschlossen worden zu sein.

Was an Zusammenhängen zwischen den Talgrundprofilen im linken Teil der Taf. II gezeichnet ist, entbehrt der stratigraphischen Gewißheit, die den meisten Lagerungsbeziehungen zwischen den Bergprofilen zuerkannt werden darf. Entscheidende Zusammenhänge in der Verzahnung zwischen Berg und Tal konnten bisher nicht aufgeschlossen, sondern nur im gedachten Vergleich erschlossen werden. Das überraschendste Ergebnis dieser Vorvergleiche ist das anscheinend hohe Alter der Schichten, die nach dem dachziegelartigen Ausstreichen jüngerer Lagen nacheinander am unteren Abhang und im Talgrund bis dicht unter die jüngste Oberflächenschicht zu liegen kommen. Ein erstaunlich hohes Alter ergibt sich vor allem für die Flußkiese des Talgrundes; sie wären, wenn irgend die in Taf. II angedeutete stratigraphische Parallelisierung richtig ist, als spätestens früh-rißeiszeitlich anzusehen. Schon seit dieser Zeit wäre unsere untere Lone zwar noch längst "gelaufen“, wahrscheinlich durchgehend oder mindestens in immer neuen, quellgespeisten Absätzen bis fast in geschichtliche Zeit; sie wäre aber doch im großen Zug der Albverkarstung schon im letzten Drittel des Eiszeitalters nicht mehr stark genug gewesen, um in großen Massen Kiese zu schütten oder auch die alten Kieslager umfassend auszuräumen. Ich kann heute nicht voraussagen, ob es technisch je gelingen wird, die entscheidende Verbindung zwischen den Profilen G. und H. der Taf. II unmittelbar sichtbar zu machen. Sie kö n n t e auch ganz anders ausfallen, als es jetzt wahrscheinlich und in der Schemazeichnung angedeutet ist - wenn nämlich nicht der ganze Schutt- 
lagenstoß von der Faustkeilkulturschicht bis zu den ockergelben tiefen Schichten in die grauen Feinschutte der distalen Profile, erstmals in Taf. II, H., einginge, sondern zu erheblichen Teilen auf dem Felsgrund ausliefe; dann wären die Talschichten einschließlich der Kiese entsprechend jünger...

Wir wollen uns aber zum Schluß daran erinnern, daß das Interesse an der zeitlichen Gliederung und Einordnung unserer Bocksteinschichten primär der Datierung der Kulturen, vor allem der Faustkeilhauptkultur der Schmiede gilt und erst sekundär die Geschichte des Lonetals und seiner Landschaft berührt. Unsere bisherigen Aufschlüsse am Bockstein stehen vorerst weitumher allein; nicht nur wir selber, auch andere mögen erst einmal um die Beschaffung vergleichbarer Befunde besorgt sein, bevor die Rückschlüsse auf die Fluß- und Landschaftsgeschichte weiter erörtert werden. Hier genüge vorerst die These, die violette Schicht des Bocksteinloches entspreche einer Phase des Riß/WürmInterglazials, die Hauptkultur der Bocksteinschmiede entweder einer frühen Phase der selben Wärmezeit oder einer Spätphase der Rißeiszeit.

Endlich darf zur Prüfung unserer zentralen Datierungsthese doch auch die kulturelle Vergleichung herangezogen werden - in dem genannten, begrenzten Umfang der gesicherten großen Linien kultureller Entfaltung und der Beziehung zwischen nächst benachbarten Profilen. Die violette Schicht dem Würm-I/II-Interstadial zuzurechnen, hieße, sie gleichzusetzen mit Schichten, in denen anderwärts jungpaläolithische Kulturen gefunden wurden („Aurignac-Schwankung“). Die violette Schicht des Bocksteinlochs führte aber eine Faustkeilkultur, die - um nur die wichtigeren, entsprechenden Kulturen des neuen Bocksteins zu nennen - der Kultur der hellbraunen Schicht, der Hauptkultur der Schmiede und der roten Schicht des Hangs zwar nicht gleich, aber doch „sippenähnlich“ ist (Abb. 1. 2. 3. 4.). Es ist äußerst unwahrscheinlich, daß eine so altertümliche Kultur einen Horizont besiedelt hätte, der anderswo von vielmals jüngeren Kulturen beherrscht war, es sei denn, man bekennte sich in pedantisch letzter Konsequenz zu der Meinung, wir hätten es in den Faustkeilen der violetten Schicht wirklich mit einer inselweis erhaltenen Reliktkultur aus längst vergangenen Zeiten zu tun.

Diesem letzten der möglichen Einwände gegen unseren Datierungsentwurf steht zunächst das morphologische Kulturbild entgegen in seiner originellen und so eindrücklich „vitalen“ und „weiterweisenden“ Formgebung. Bei aller Subjektivität derartiger, rein morphologischer Beurteilung kann ihnen doch nicht jede Berechtigung abgesprochen werden - vollends, wenn sie nur zur Ergänzung anderer Argumente und nicht für sich allein gewertet werden. Weiterhin rechnen wir zwar immer mit kulturellen Überschneidungen zwischen abgehenden und aufsteigenden Kulturformen; unsere eigenen Grabungen im Lonetal haben den Anlaß zu konkreter Erwägung einer solchen Überschneidung gegeben (Völzing, Fundberichte aus Schwaben N.F. IX, 1938). Aber ihre Spanne wäre denn doch $\mathrm{zu}$ weit gestreckt, wollten wir die gleichzeitige Existenz echter Faustkeilkulturen und eindeutig jungpaläolithischer Aurignacienkulturen postulieren. Handelt es sich doch um eine ganze "Sippe“ von Faustkeilkulturen am neuen Bockstein, um eine, über mindestens nicht allzu kurze Zeit hinweg blühende, lebendig sich verändernde Kulturengruppe; sie kann nicht wohl als jener Spätling gelten, der ja dann auch die ganzen zwischenliegenden Epochen der "Moustérienkulturen" verschlafen haben müßte.

Es kommt hinzu, daß am alten Bockstein schöne und reiche Aurignacienkulturen wirklich gefunden wurden (Bürger, Mitteilungen des Vereins für Kunst und Alterthum in Ulm und Oberschwaben 1892; WetzeL, Mitteilungen des Vereins für Naturwissenschaft und Mathematik in Ulm 24, 1954). Sie liegen in lößig-lehmigen Kalkschuttschichten, die nach dem stratigraphischen Vergleich (unmittelbare Zusammenhänge sind nicht aufgeschlossen) dem oberen Löß der Bocksteinschmiede entsprechen dürfte. Aber auch der darunter folgende, rötliche Lehm führt noch Aurignacien mit nicht immer typischem Steinwerkzeug und mit schönen Knochenspitzen. Sollte die noch nicht vor- 

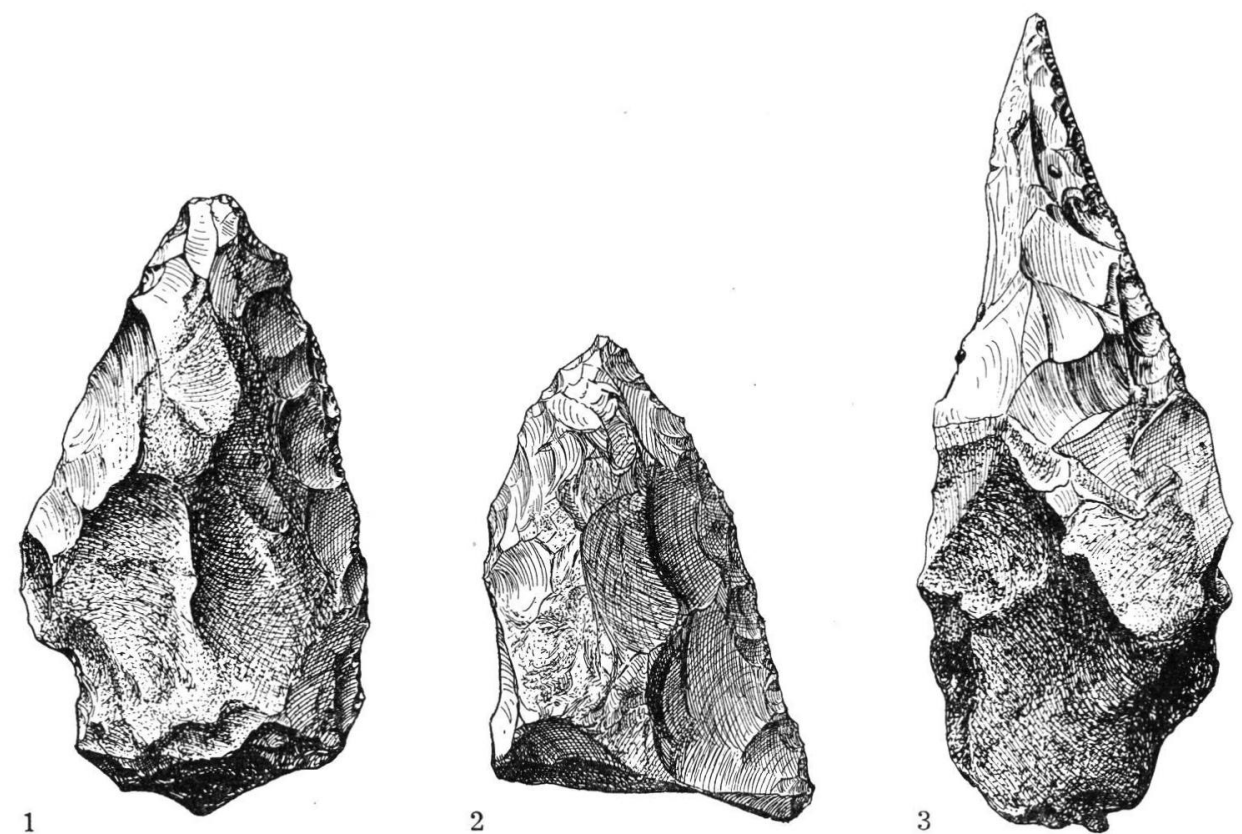

Abb. 1. Faustkeil 3264 aus der violetten Kulturschicht des Bocksteinlochs, 6. 9. 1934. 2/3 nat. Gr. Abb. 2. Faustkeil 3210 aus der hellbraunen Kulturschicht des Bocksteinlochs, 4. 9. 1934. 2/3 nat. Gr. Abb. 3. Faustkeil 2145 aus der Hauptkultur der Bocksteinschmiede, 1. 9. 1933. 2/3 nat. Gr.
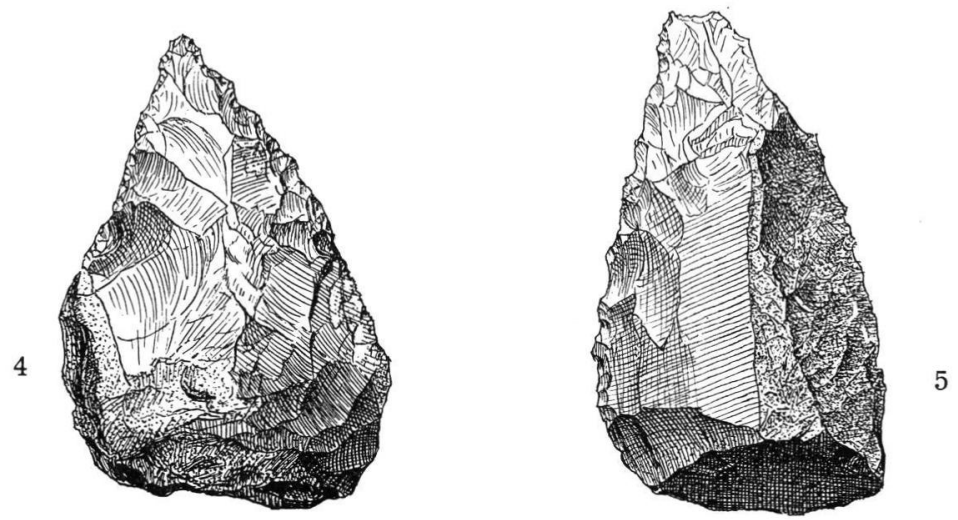

Abb. 4. Faustkeil 5558 aus der roten Schicht des Abhangs, 19. 8. 1955. 2/3 nat. Gr. Abb. 5. Faustkeil T 882 aus dem tiefen Lehm am Törle der alten Bocksteinhöhle, 6. 9. 1956. $2 / 3$ nat. Gr.

liegende Sedimentanalyse dieser rötlichen Lehmschicht den Anhalt für eine Wärmeschwankung ergeben, so wäre stratigraphisch dazu auf ihre, zum feinsteinigen Lehm der Schmiede parallele Lage zu verweisen. Während am neuen Bockstein keine Spur von Aurignacien zu finden war, haben die, seit 1955 unter der mitverantwortlichen Mitarbeit von Marie Luise WIRsING weitergeführten Grabungen am Törle der alten Bocksteinhöhle etliche „bocksteintypische" Faustkeile ergeben (Abb.5), und zwar aus den gelblichen Lehmen, die in klarer Scheidung unter den tiefsten Lagen der Aurignacienhorizonte folgen. Damit ist aber auch am Bockstein die große Linie der Kulturenfolge 
bestätigt, die - von aller Frage nach den eigentlichen Moustérienkulturen abgesehen uns nur soweit wichtig ist, als ganz gewiß auch hier am alten, aber auch gültig für den neuen Bockstein, die Faustkeilkulturen älter sind als die jungpaläolithischen Klingenkulturen mit Knochenwerkzeugen. Die Kultur der violetten Schicht des Bocksteinlochs kann nicht zur Zeit einer "Aurignacien-Schwankung“ bestanden haben; die Frage, welcher noch unbekannten oder schon bekannten Faustkeilkulturnuance die Faustkeilfunde am alten Bockstein zuzurechnen seien, braucht hier nicht berührt zu werden.

Alle, aus kulturellen Vergleichen zusätzlich zu gewinnenden Anhalte bestätigen somit, daß die violette Schicht zu einer Phase des klassischen Riß/Würm-Interglazials gehört, daß also die Hauptkultur der eigentlichen Bocksteinschmiede noch älter ist.

Aus dem Ergebnis gleichlaufender stratigraphischer, sedimentanalytischer und floristischer, dann und wann auch faunistischer Befunde gewinnen wir die Berechtigung, in der eigenständig eigenartigen Kultur der Schmiede wirklich, wie dies der unbefangenen Betrachtung von vornherein erscheinen mußte, eine „Grundkultur“ zu sehen und nach den, in ihr enthaltenen Ansätzen zu weiterführender Entfaltung zu fahnden.

Manuskr. eingeg. 6. 5. 1957.

Anschrift des Verf.: Prof. Dr. Robert Wetzel, Tübingen, Waldhäuserstraße 16. 